\title{
Stereological and Morphometric Study of Type 3 Collagen Formation in the Cutaneous Wounds of Diabetic Mice Treated with Mesenchymal Stem Cells
}

\author{
Huanna Waleska Soares Rodrigues', Betânia Souza Monteiro², Lucilene dos Santos Silva', \\ Maria Acelina Martins de Carvalho' \& Napoleão Martins Argôlo Neto'
}

\begin{abstract}
Introduction: Wound healing is a progressive, essential and complex physiological process that occurs as a restorative response after a tissue injury. It involves three phases: inflammation, proliferation and maturation. Exogenous, endogenous and pathological factors may interfere in the cicatricial process in humans and animals by altering the balance between the synthesis, degradation and remodelling of collagen and elastic fibres. Diabetes mellitus is a progressive metabolic disease that alters elastogenesis and collagenesis and induces delays in the healing process. Scientific evidence suggests that mesenchymal stem cells modulate the cicatricial response. Thus the objective of this work was to perform stereological and morphometric analysis to determine the formation of dermal fibres in cutaneous fragments of a murine model of diabetes mellitus.

Materials, Methods \& Results: Histological sections were obtained from the cutaneous wounds of diabetic mice. The cutaneous wounds were previously treated with autogenous mesenchymal stem cells, physiological solution or polyurethane membrane. The histological sections were subsequently processed and stained for type 1 and 3 collagen fibres and elastic fibres using Picrosirius Red and Weigert staining, respectively. Histological sections stained with Picrosirius Red presented three types of birefringence under polarised light microscopy that corresponded to red colours for type 1 collagen and green and yellow colours for type 3 collagen. Weigert staining presented three colours for histological structures under white light microscopy that corresponded to black colours for elastic fibres, variations in colour from pink to purple for other structures and dermal attachments. The elastic fibres, represented by a black colour, presented in a heterogeneous form and were either identified as thin, punctiform or rectangular fibres or as elastic agglomerates. A greater volume of elastic fibres was observed in the superficial dermis than in the deep dermis, arranged irregularly. These fibres were organised longitudinally to the dermo-epidermal junction and surrounding the blood vessels and hair follicles. The images obtained were evaluated using the Cavalieri principle of stereology to obtain quantitative data in three-dimensions (3D), represented by the volume of the dermal fibres, and by the colour segmentation method. The K-means clustering plug-in in Image ${ }^{\circledR}{ }^{\circledR}$ was used to quantify the area of the dermal fibres in the cutaneous wounds after the proposed dermatological treatments. A total of 90 images were obtained and analysed. No statistically significant differences $(P>0.01)$ were observed in the volume or area of type 1 collagen fibres between the treatment groups. Significant differences $(P<0.01)$ were only identified for the volumes and areas of type 3 collagen, with treated animals also presenting lower mean values for the volume and area of elastic fibres compared to the control group.

Discussion: The preponderance of type 3 immature collagen in the cutaneous wounds of animals treated with stem cells indicates active collagenase and greater fibroblastic activity, which is probably induced by stem cells. Diametrically, the identification of lower levels of elastic fibres in the cutaneous fragments treated with stem cells suggests that cell therapy does not contribute satisfactorily to elastogenesis. Previous reports suggested that mesenchymal stem cells may decrease elastin synthesis, and such a situation may have occurred in this study. The autologous mesenchymal stem cells increased the formation of collagen fibres in diabetic mice at the detriment of the formation of elastic fibres, thus suggesting active early collagen in the first 2 weeks of the cicatricial process.
\end{abstract}

Keywords: stereology, mesenchymal stem cells, healing, collagen. 


\section{INTRODUCTION}

Wound healing is a progressive, essential and complex physiological process that occurs as a restorative response after a tissue injury. It involves three phases: inflammation, proliferation and maturation and can be influenced by local or systemic factors [22]. However, scarring of aberrant wounds is also associated with underlying processes, including diabetes, cancer and malnutrition. Of the major concerns of chronic wounds, perhaps none is as threatening or as important as diabetes mellitus, which leads to changes in the physical and functional structure of the skin [11].

Evidence of using mesenchymal stem cells (MSC) to treat patients with diabetes is promising $[2,15]$. Preclinical studies have demonstrated some of the skilful characteristics of MSC, including their immunomodulatory capacity, their ability to stimulate angiogenesis and reduce scars and their high therapeutic potential in regenerative medicine $[13,19,20]$. Although MSC may promote a systemic improvement in the lesion and accelerate the cicatricial process of diabetic wounds, the impact of these cellular treatments on the formation of elastic and collagen fibres, which are essential for wound closure, has rarely been studied.

Thus the objective of this study was to verify the formation of dermal fibres in cutaneous fragments from diabetic animals treated with MSC. Analysis and image processing was used to obtain quantitative data in threeand two-dimensional (3D) elements in repaired tissues.

\section{MATERIALS AND METHODS}

Samples

Biopsy specimens were kindly provided by Argôlo Neto et al. [3] who previously studied the effect of autogenous treatment with MSC and platelet-rich plasma on the cutaneous healing of diabetic mice.

A total of 18 donor cutaneous fragments embedded in histological paraffin were used. These samples were used to represent the cutaneous healing process in 3-week-old male mice (Mus musculus) with streptozotocin-induced diabetes mellitus. The 18 cutaneous fragments were split between three experimental groups with six animals in each. Animals were euthanised on the 12th day after treatment, and an elliptical excisional biopsy was performed to remove the cutaneous tissue involved in the healing process and a small margin of adjacent skin [3].
Of the 18 donor samples, six corresponded to wounds treated with daily cleaning using a sterile physiological solution (control group- GCL), six corresponded to wounds treated daily with a sterile saline solution and covered with a semipermeable adherent polyurethane membrane (membrane group - GME) and six corresponded to wounds treated with autogenous transplantation of bone marrow-derived MSC (3.3 x 10 cells $/ \mathrm{mL})$ (GCT) in a single transsurgical application and covered with a semipermeable adherent polyurethane membrane to retain the stem cells in the lesional bed [3].

Stereology

Stereology applications were adopted in this study in order to obtain accurate and unbiased quantitative data. A total of 90 histological sections were obtained and subjected to routine histological processing. The sampling method employed was vertical, uniform, systematic and randomised due to the layered structure of the skin [14]. The 18 paraffin blocks were submitted to serial cuts of $4 \mu \mathrm{m}$ thickness using a rotary microtome (Leica Biosystems RM2265) ${ }^{1}$. The cuts were perpendicular to the epidermal surface and, therefore, parallel to the vertical axis.

Consecutive histological sections from each block were stretched on glass slides, and one cut from every 10 was selected to ensure a thickness of $40 \mu \mathrm{m}$ between histological sections. Picrosirius Red, under polarised light microscopy (Vertical Microscope Led Leica DM2700 M) ${ }^{1}$, and Weigert staining were subsequently used to identify type 1 and 3 collagen fibres and elastic fibres, respectively. Photomicrographs (10x magnification) of transverse sections were obtained from each slide using a microscope system (Olympus CX33 Biological Microscope $)^{2}$ coupled to a digital video camera (Digital Camera Sony Cyber Shot DSC-HX400V 20.4 Megapixels) ${ }^{3}$ and a microcomputer (Positive Stilo DS3515 com Intel ${ }^{\circledR}$ Dual Core, 2GB, 320GB $)^{4}$.

Image $J^{\circledR 5}$ software was used to evaluate the photomicrographs and quantitatively determine the three-dimensional parameters of the dermal fibres (collagen and elastic) from the two-dimensional sections stained with Picrosirius Red and Weigert. During the stereological analysis, the Grid plugin was used to apply the test system (or quadrant mesh) of points in the digital photomicrographs. An initial 300-point test system was used to empirically determine the volume density $(\mathrm{Vv})$ using $\mathrm{Vv}=\mathrm{P}_{\mathrm{p}} / \mathrm{P}_{\mathrm{t}}$, where $\mathrm{P}_{\mathrm{p}}$ was the number 
of points in the collagen or elastic fibres and $\mathrm{P}_{t}$ was the number of total points in the test system [1]. After establishing the Vv in each sample, the number of sample points (n) required in the test system with a relative standard error (e) of 0.05 was calculated by the formula: $\mathrm{n}=1-\mathrm{Vv} /(\mathrm{e})^{2}$. The mean values were determined for each experimental group, and the highest mean was chosen among the evaluated ones, adopting e $=0.05$ which corresponded to the $95 \%$ confidence interval.

After the quantification of points, the Cavalieri principle was used. This principle states that the total volume of a structure can be estimated from the product of the distance between the sections of systematic and random intervals and the sum of the areas of the object [14]. The area of the object (Aob) was acquired by counting areas of interest for each dermal fibre (collagen and elastic) using the test system superimposed on the photomicrograph. Each point of the object $(p t)$ was associated with the area of the point (apt), with Aob obtained by the formula: Aob $=\Sigma p t x$ apt.

The volume, according to the Cavalieri principle, was measured using Aob using the following formulas for different variables: $V t$ [ col 1] $=\Sigma p t$ [coll] $x$ apt $x T$; Vt [col3] $=\Sigma p t[\operatorname{col} 3] x$ apt $x T$; Vt[fe $]=$ $\Sigma p t[f e] x$ apt $x T$, where $p t$ was the points that affected the type 1 [coll] and 3 [col3] collagen and elastic fibres $[f e]$ in each evaluated cut, apt was the area associated with the point defined by the test system and $T$ was the known thickness between histological sections $(40 \mu \mathrm{m})$. The total volume $(V t)$ of each analysed structure was expressed in $\mathrm{mm}^{3}$.

\section{Morphometric analysis}

The colour intensity image segmentation method was used for the two-dimensional analysis. The Picrosirius Red- and Weigert-stained photomicrographs were standardised in contrast using Image $\mathbf{J}^{\circledR}$.

To measure the real area of the dermal fibres (collagen and elastic) in $\mathrm{mm}^{2}$, pixels were converted into mm using Image $\mathrm{J}^{\circledR}$ with a value of 250 pixel $/ 1 \mathrm{~mm}$. The routine menu function analyse $>$ measure was used. Images were then segmented in colour using the K-means clustering algorithm [18], which automatically grouped pixels according to colour.

The digital photomicrographs showing Picrosirius Red staining were used to identify the most representative classes or clusters for measuring the area of type 1 and 3 collagen fibres. Three clusters were automatically selected that corresponded to type 1 collagen (red colour), type 3 collagen (yellowish-green colour) and the background (black colour). The digital photomicrographs showing Weigert staining were segmented into four colour clusters that corresponded to elastic fibres (black), other structures (pink), dermal attachments (purple) and the background (white). The selection of each cluster was performed using the threshold method in the colour threshold plug-in (Image $>$ Adjust $>$ Color Therhsold) in Image $\mathbf{J}^{\circledR}$. The RGB colour system was used to estimate the real image area of each selected skin fibre in the segmentation process.

\section{Statistical analysis}

Statistical analysis was performed using Bioestat $^{\circledR}$ software v.5.9. Analysis of variance (ANOVA) was used to identify statistically significant differences between the independent groups, while Tukey's average comparison test was used when a treatment effect was detected. A rejection level of the null hypothesis of was adopted for both analyses.

\section{RESULTS}

Histological sections stained with Picrosirius Red presented three types of birefringence under polarised light microscopy that corresponded to red colours for type 1 collagen and green and yellow colours for type 3 collagen (Figure 1). Weigert staining presented three colours for histological structures under white light microscopy that corresponded to black colours for elastic fibres, variations in colour from pink to purple for other structures and dermal attachments (Figure 2). The elastic fibres, represented by a black colour, presented in a heterogeneous form and were either identified as thin, punctiform or rectangular fibres or as elastic agglomerates. A greater volume of elastic fibres was observed in the superficial dermis than in the deep dermis, arranged irregularly. These fibres were organised longitudinally to the dermo-epidermal junction and surrounding the blood vessels and hair follicles. Variations in colour from pink to purple were correlated to fibroblasts, collagen, sebaceous glands and hair follicles (Figure 2).

For the for calculation of the volume of type 1 (VCOL1) and 3 (VCOL3) collagen fibres, a total of 48 points was the number of sample points (n) required for the test system with $\mathrm{e}=0.05$ in a point area of 1.136 $\mathrm{mm}^{2}$. For the evaluation of elastic fibres (VEF), a total of 70 points was the number of sample points (n) required with $\mathrm{e}=0.05$ in a point area (apt) of $0.40 \mathrm{~mm}^{2}$. 
During stereological analysis, no significant differences $(P>0.01)$ were observed in the mean volume of type 1 collagen (VCOL 1 ) between the different treatment groups. In contrast, there were significant differences $(P<0.01)$ between type 1 and 3 collagen in each experimental group, with the highest averages identified for VCOL1 (Table 1).

Significant differences $(P<0.01)$ were also identified in the volumes of type 3 collagen (VCOL1) between animals treated with the polyurethane adherent membrane (GME) and those treated with autologous MSC (GCT). The highest averages were observed in the GCT group when compared to the other treatment groups. No significant differences $(P$ $>0.01$ ) were observed for this variable between the control (GCL) and GME groups or between the GCL and GCT groups. The lowest means were observed in the GME group followed by the GCL group (Table 1).

In the histological analysis, a higher deposition of type 3 collagen fibres (characterised by yellow and green colours) was identified in animals treated with autologous MSC compared to the other treatment groups. In contrast, animals treated with the polyurethane adherent membrane presented intermediate deposition of VCOL3 compared to the GCT group. The GCL group had the lowest deposition of type 3 collagen fibres (Figure 3).

Stereological evaluation of the elastic fibre volume (VFE) identified significant differences $(P<$ $0.01)$ between the different treatment groups. Animals in the GCT group had the lowest mean values for VFE, while the GCL group presented the highest averages when compared to the other groups (Table 1).

In contrast to the stereological analysis of the Picrosirius Red-stained sections, only two relevant clusters corresponding to red colours for collagen type 1 and yellow for collagen type 3 were identified in the morphometric analysis by colour segmentation (Figure 4). Similarly, Weigert staining only revealed one relevant cluster, which corresponded to black for elastic fibres, under light microscopy (Figure 5).

A progressive segmentation of colours was obtained through the K-mean clustering algorithm (which identifies the colour cluster of the collagen fibre) and the colour threshold plug-in (which selects that cluster). Type 1 collagen fibres were identified in white (Figure 6A) and the fibre contour was identified in blue (Figure 6B), thus delimiting the area of the type 1 collagen fibres (ACOL1). During the thresholding process, Image $\mathrm{J}^{\circledR}$ software enhanced in red the type 1 collagen fibres outlined in blue. The segments stained in yellow corresponded to type 3 collagen fibres (Figure 6B). Similarly, type 3 collagen fibres were initially identified in white (Figure 6C) and the fibre contour was identified in blue (Figure 6D), thus delimiting the area of the type 3 collagen fibres (ACOL3). During the thresholding process, Image $\mathrm{J}^{\circledR}$ emphasised in yellow the type 3 collagen fibres outlined in blue (Figure 6D).

No significant differences $(P>0.01)$ were observed between the groups for the variable area of type 1 collagen; however, significant differences $(P$ $<0.01$ ) between the ACOL1 and ACOL3 areas were identified in each treatment group, with the highest averages observed in ACOL1 (Table 2). The type 3 collagen area increased significantly $(P<0.01)$ in the group treated with autologous MSC (GCT) compared to the control group. No significant increase $(P>$ $0.01)$ was observed for the ACOL3 variable between the GCL and GME groups or between the GCT and GME groups (Table 2).

For the colour segmentation analysis of the histological sections stained using the Weigert technique, elastic fibres were identified in red (Figure 7A) and the fibre contours were identified in blue (Figure 7B), thus delimiting the area of the elastic fibres (AFE).

A significant difference $(P<0.01)$ was identified between the groups for the AFE variable. The GCL group had the largest area of elastic fibres, followed by the GME and GCT groups, respectively (Table 2).

\section{DISCUSSION}

The birefringent colour spectrum observed in Picrosirius Red staining, as red for type 1 collagen and green and yellow for type 3 collagen, as well as the identification of black elastic fibres in Weigert staining, are well documented in the literature [16,17]. According to these authors, type 1 collagen corresponds to the mature form of the fibre while type 3 collagen corresponds to the immature form; however, both types of fibre were observed to be intertwined in the histological sections evaluated, thus denoting the process of maturation of the fibres in progress (Figure 3).

Weigert colouration allows identification of the elastic system, which is composed of immature (except oxitalanic fibres) and mature fibres [16]. The current study denominated elastic fibres as those 
H.W.S. Rodrigues, B.S. Monteiro, L.S. Silva, M.A.M. Carvalho \& N.M. Argôlo Neto. 2018. Stereological and Morphometric Study of Type 3 Collagen Formation in the Cutaneous Wounds of... Acta Scientiae Veterinariae. 46: 1553.

Table 1. Mean stereological values of the volume of collagen (VCOL1 and VCOL3) and elastic fibres (VFE) in healing wounds in C57BL/6 diabetic mice $(\mathrm{n}=18)$.

\begin{tabular}{cccccccc}
\hline Variable $(\mathrm{mm} 3)$ & GCL & $\%$ & GME & $\%$ & GCT & $\%$ & $P$ \\
\hline VCOL1 & $0.6513 \pm 0.22^{\mathrm{a}}$ & 75.2 & $0.5513 \pm 0.20^{\mathrm{a}}$ & 76.8 & $0.4725 \pm 0.20^{\mathrm{a}}$ & 58.9 & 0.08 \\
VCOL3 & $0.2150 \pm 0.14^{\mathrm{bc}}$ & 24.8 & $0.1666 \pm 0.10^{\mathrm{b}}$ & 23.2 & $0.3301 \pm 0.12^{\mathrm{c}}$ & 41.1 & 0.002 \\
VFE & $0.3242 \pm 0.05^{\mathrm{d}}$ & - & $0.2368 \pm 0.04^{\mathrm{e}}$ & - & $0.1728 \pm 0.03^{\mathrm{f}}$ & - & 0.0001 \\
\hline
\end{tabular}

GCL: control treatment; GCT: treatment with autologous mesenchymal stem cells; GME: treatment with a polyurethane membrane. Means followed by different letters in the same column differed by $1 \%$ significance in Tukey's comparison test.

Table 2. Mean morphometric values of the area of collagen (ACOL1 and ACOL3) and elastic fibres (AFE) in the healing skin wounds of C57BL/6 diabetic mice $(\mathrm{n}=18)$.

\begin{tabular}{cccccccc}
\hline Variable $(\mathrm{mm} 2)$ & GCL & $\%$ & GME & $\%$ & GCT & $\%$ & $P$ \\
\hline ACOL1 & $5.367 \pm 2.7^{\mathrm{a}}$ & 78.99 & $5.935 \pm 2.7^{\mathrm{a}}$ & 67.88 & $5.937 \pm 1.51^{\mathrm{a}}$ & 60.07 & 0.7571 \\
ACOL3 & $1.427 \pm 0.99^{\mathrm{b}}$ & 21.01 & $2.808 \pm 0.95^{\mathrm{bc}}$ & 32.12 & $3.945 \pm 1.76^{\mathrm{c}}$ & 39.93 & 0.0001 \\
AFE & $0.3242 \pm 0.05^{\mathrm{d}}$ & - & $0.2368 \pm 0.04^{\mathrm{e}}$ & - & $0.1728 \pm 0.03^{\mathrm{f}}$ & - & 0.0001 \\
\hline
\end{tabular}

GCL: control treatment; GCT: treatment with autologous mesenchymal stem cells; GME: treatment with a polyurethane membrane. Means followed by different letters in the same column differed by $1 \%$ significance in Tukey's comparison test.

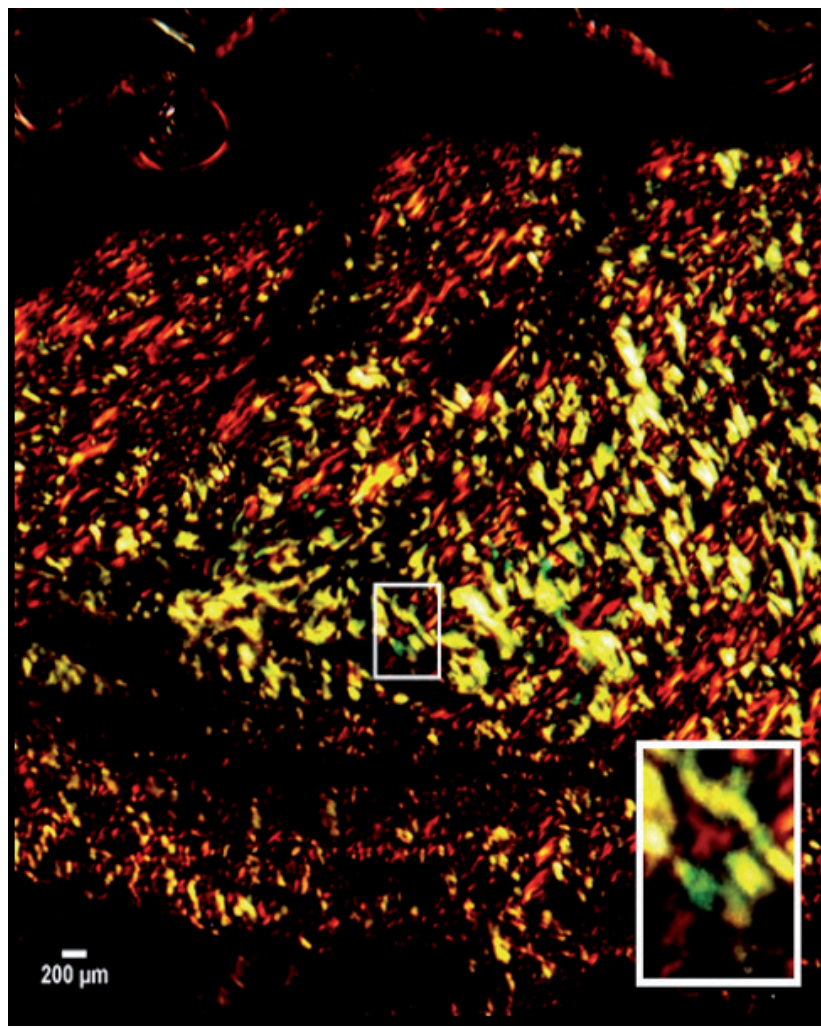

Figure 1. Photomicrograph of the healing area of a cutaneous wound in a diabetic mouse treated with MSC. Zoom detail for the observation of collagen type 1 fibres with red birefringence and type 3 collagen with green and yellow birefringence. Picrosirius Red under polarised light [10x magnification].

structures stained in black; however, such staining corresponded to both elauninic (immature) and elastic (mature) fibres [21]. Significant morphological heterogeneity of elastic fibres was identified between the different treatment groups, thus suggesting that such variability represents the difference between mature

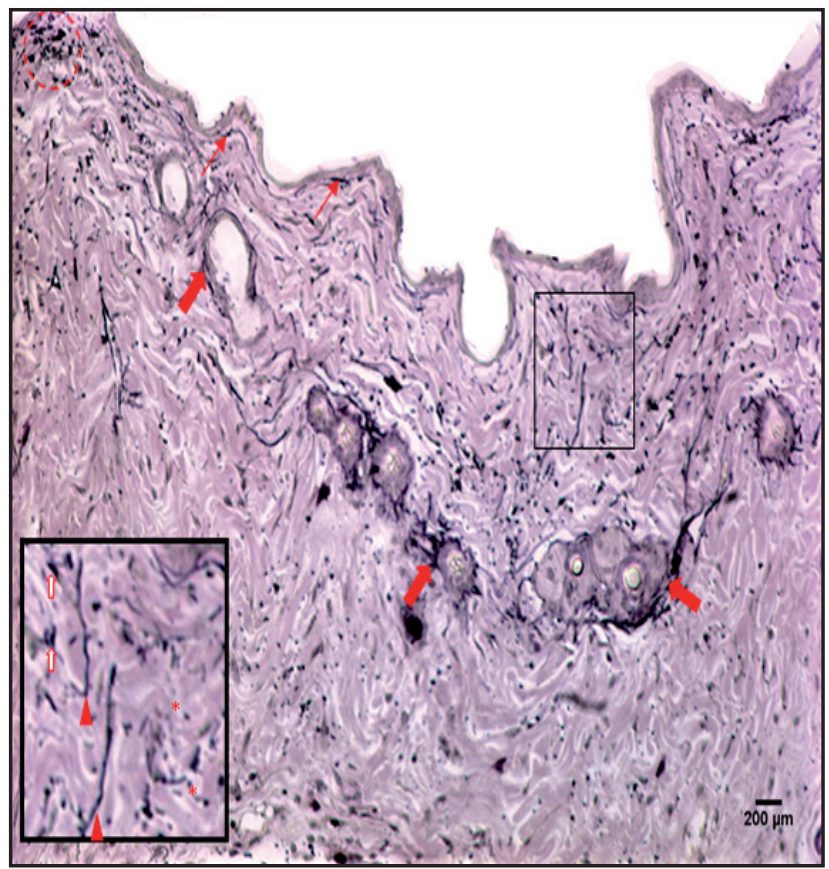

Figure 2. Photomicrograph of the healing area of a cutaneous wound in a diabetic mouse treated with MSC. The elastic fibres are organised longitudinally to the dermo-epidermal junction (thin arrow) and circumferentially to the blood vessels and hair follicles (thick arrow). Zoom detail for the observation of elastic fibres in black colouration. Morphological variability of the elastic fibres was observed as thin (arrowhead), punctiform (asterisk), short rectangular profile (hollowed arrow) and agglomerated elastic (dotted circle) fibres. Weigert under white light [10x magnification].

and immature fibres, as well as the three-dimensionality of these fibres, arranged in the dermis (Figure 2). The colour variations (pink to purple) observed in the Weigert colouration corresponded to fibroblasts, collagen, sebaceous glands and hair follicles stained with haematoxylin [8]. 


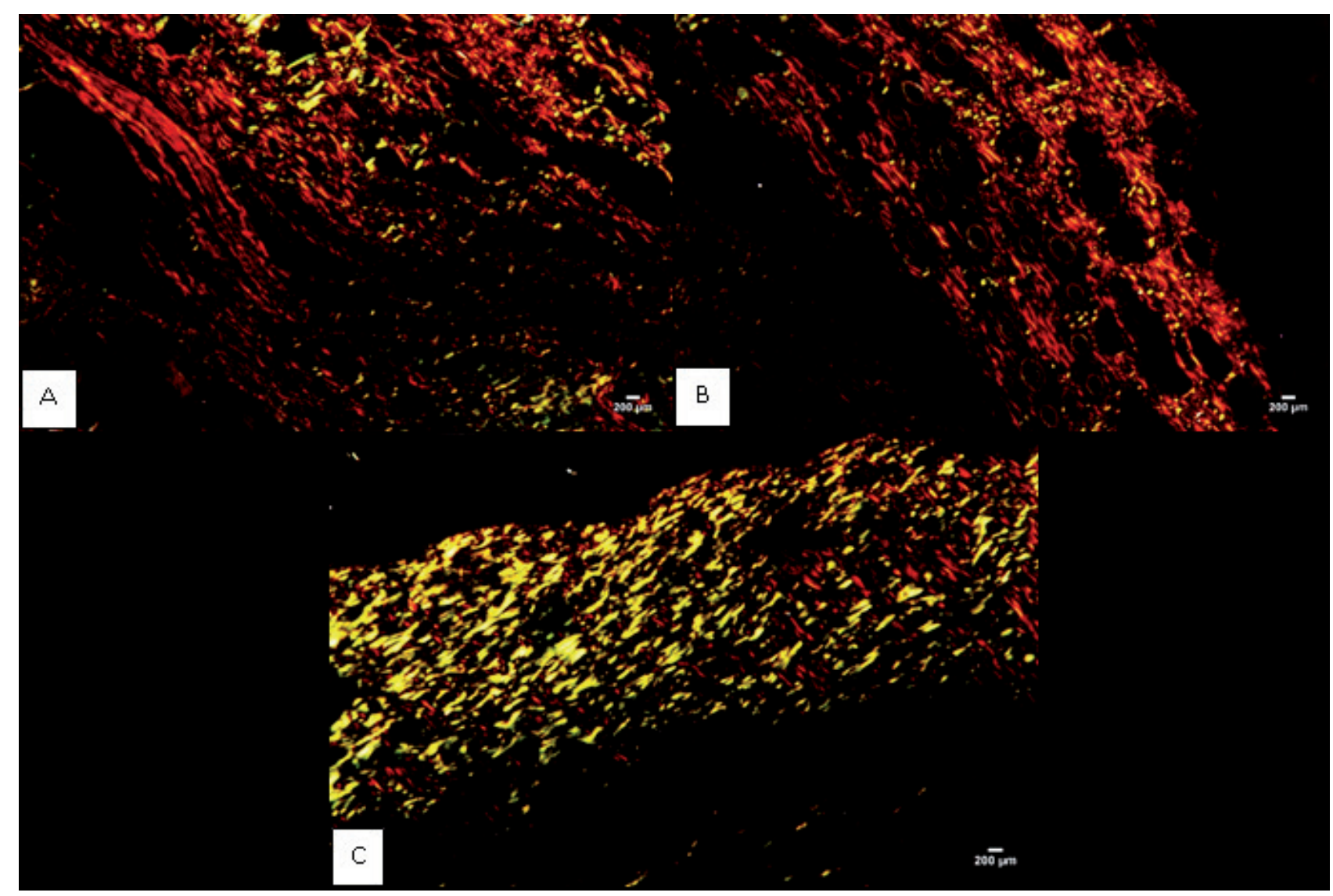

Figure 3. Photomicrographs of the healing areas of cutaneous wounds in diabetic mice after 12 days of evolution. A- Control group (GCL) animal with reduced type 3 collagen (VCOL3) deposition compared to the group treated with autologous MSC (GCT). B- Animal from the group treated with an adherent polyurethane membrane (GME) with lower deposition of VCOL3 than the GCL group. C- GCT group with intense deposition of type 3 collagen fibres in comparison to the other groups. Picrosirius Red [10x magnification].

The determination of the 48 and 70 sample points required for the test system with $\mathrm{e}=0.05$ to calculate the volume of collagen and elastic fibres, respectively, was a mandatory step for the stereological analysis. In addition to reducing the working time and the number of images required for the analysis, it also provides reliable data by subordinating them to a 95\% confidence interval [1].

No significant variation was identified in the mean volume of type 1 collagen between the different treatment groups (Table 1), which corroborates contemporary studies that indicate that this is a common physiological characteristic among species $[9,23,28]$. Type 1 collagen represents more than $80 \%$ of collagen fibres in humans, mice and dogs, whereas type 3 collagen represents only $10 \%$.

An increase in collagen degradation is usually detected in humans or animals with diabetes. This occurs due to the release of metalloproteinases from dendritic cells activated by the nonspecific inflammatory response or by the oxidative action of advanced glycation end products on collagen fibres [6]. The volume of type 1 collagen was not measured before the induction of diabetes mellitus in this study; however, it is believed that at only 12 days after the cicatricial process, type 3 collagen matures into type 1 collagen, which was probably reflected in the maintenance of the mean volume of mature fibres between the treatment groups. These findings agree with previous descriptions that the maturation of dermal collagen fibres intensifies from 3 weeks after the beginning of the cicatricial process [27,31].

In contrast, a significant difference was found between the mean volumes of type 1 and type 3 collagen in the different treatment groups (Table 1), with a predominance of the latter among animals treated with autologous MSC. The syllogism that persisted active neocollagenesis in the GCT group by increasing the volume of immature fibres was assumed. Under physiological conditions, neocollagenesis starts from the third day of the cicatricial process and ends at the end of the second week when there is greater maturation and remodelling of the collagen fibres [27]. The volume of these fibres is expected to reduce in diabetic 


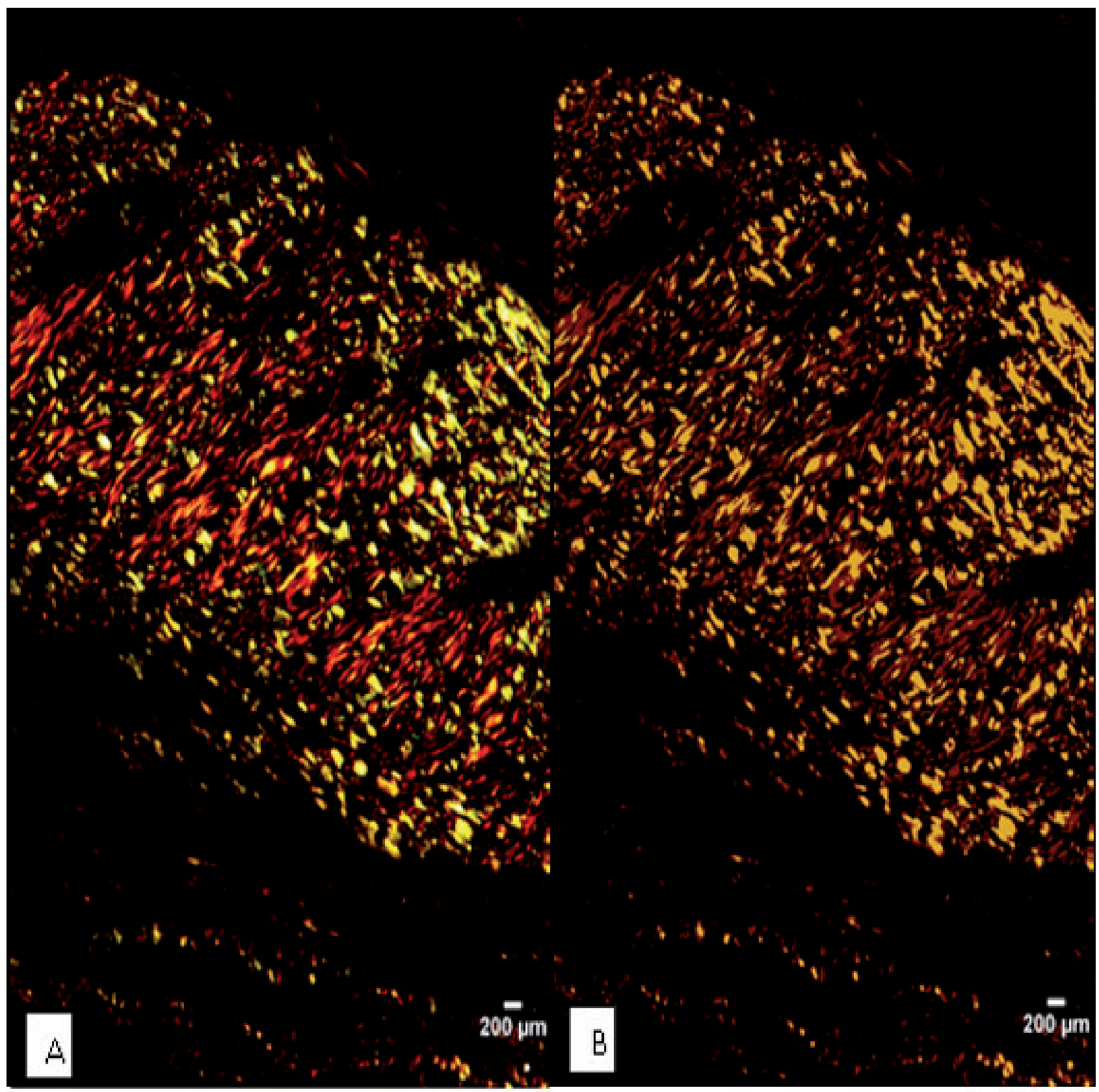

Figure 4. Photomicrographs of the healing area of cutaneous wounds in diabetic mice treated with MSC. A- Original image without analysis using targeting cluster colour. B- Photomicrograph obtained by analysing the K-mean clustering algorithm, which expresses the type 1 collagen fibres in red and the type 3 collagen fibres in yellow. Method of colour segmentation. Picrosirius Red under polarised light [10x magnification].

patients due to the increased fibre degradation induced by the disease [6]. Nevertheless, the indirect observation of late neocollagenesis in the cicatricial process highlights the relevance of MSC treatment for the synthesis of collagen in the proposed model.

Several contemporary studies have shown that MSC positively contribute to cutaneous healing by reducing the lesional area, reducing the wound healing time, increasing granulation tissue formation and increasing collagen synthesis in humans and animals $[14,19]$. MSC secrete numerous paracrine factors (i.e. vascular growth factor, insulin-like growth factor, stromal cell growth factor etc.) that induce chemotaxis of fibroblasts and keratinocytes, inhibit apoptosis, promote angiogenesis and stimulate neocollagenesis $[13,14,19,20]$. Although the production of these bioactive peptides was not measured in this study, it is believed that this situation could have occurred among animals in the GCT group.

The intense deposition of type 3 collagen in animals in the GCT group was histologically evident from the colouring of Picrosirius Red (Figure 5). This was 


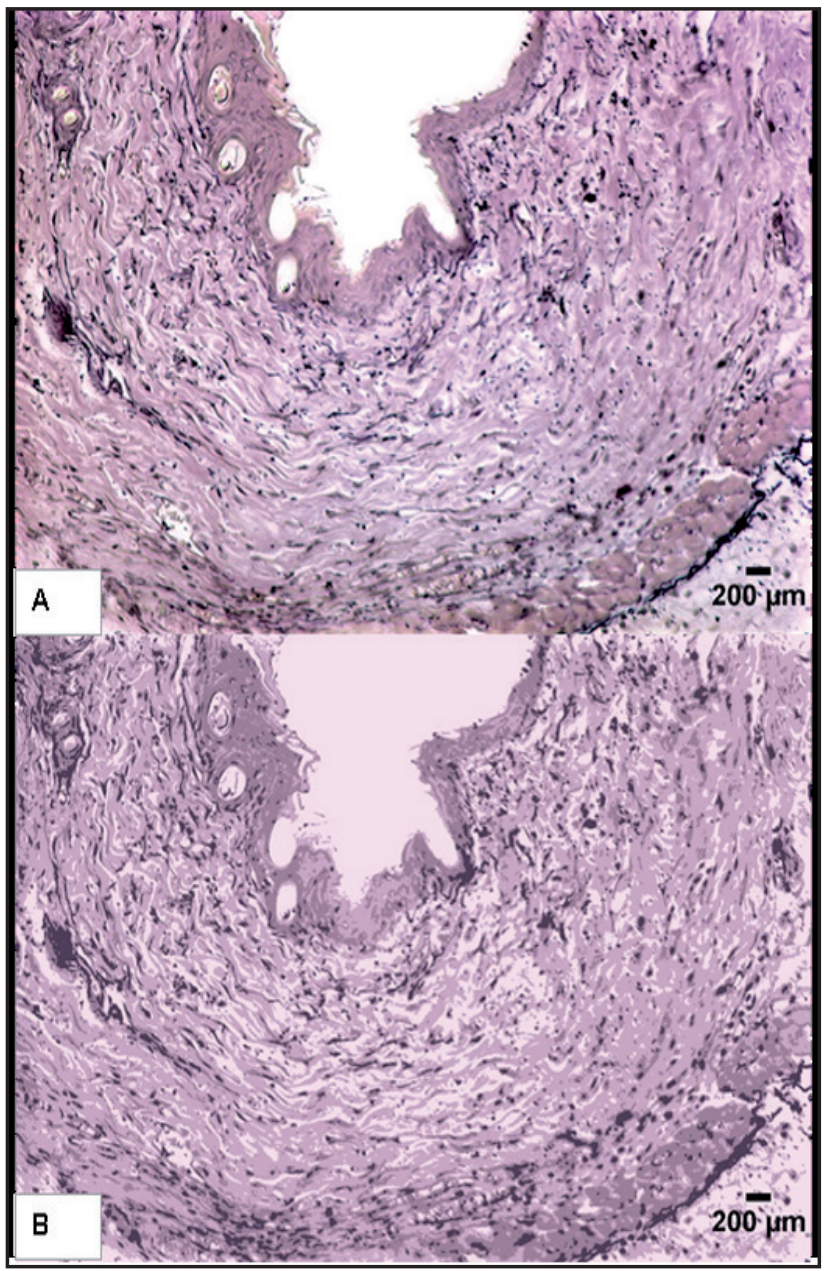

Figure 5. Photomicrographs of the healing area of cutaneous wounds in diabetic mice treated with MSC. A- Original image without analysis by targeting cluster colour, with observed variation in the spectrum of shades of black. B- Image with colour segmentation, showing elastic fibres identified in the unique black spectrum. Weigert [10x magnification]. also observed in the GCL and GME groups where the red colour predominated relative to type 1 collagen and gave rise to the premise of low collagen in these groups.

The elastic fibre volume was not evaluated before the induction of diabetes mellitus; however, as described in previous studies [24,31], it is believed that the progression of the disease contributes to the increased degradation of these fibres. Elastases and metalloproteinases were suggested to be continuously expressed in active proteolytic active tissues, such as chronic wounds, thus inducing continuous degradation of mature elastic fibres and resulting in elastolysis superior to elastogenesis. Despite the results obtained for collagen fibres, there was no increase in the elastic fibre deposition in the cutaneous lesions of animals treated with MSC (Table 1).

Recent studies showed that MSC may decrease elastin synthesis or increase the degradation of existing elastic fibres [7,31]. The molecular mechanisms that guide these events remain misunderstood, and such hypotheses are in conflict with previous descriptions that demonstrated a positive contribution of MSC treatment for elastolytic disorders such as aortic aneurysms or chronic obstructive pulmonary disease [29]. In addition, one recent study showed that the MSC secretome inhibited dermal elastic matrix degradation [31].

Despite the previous data, the data in this study suggested that the late active neocollagenesis induced by MSC treatment delayed the onset

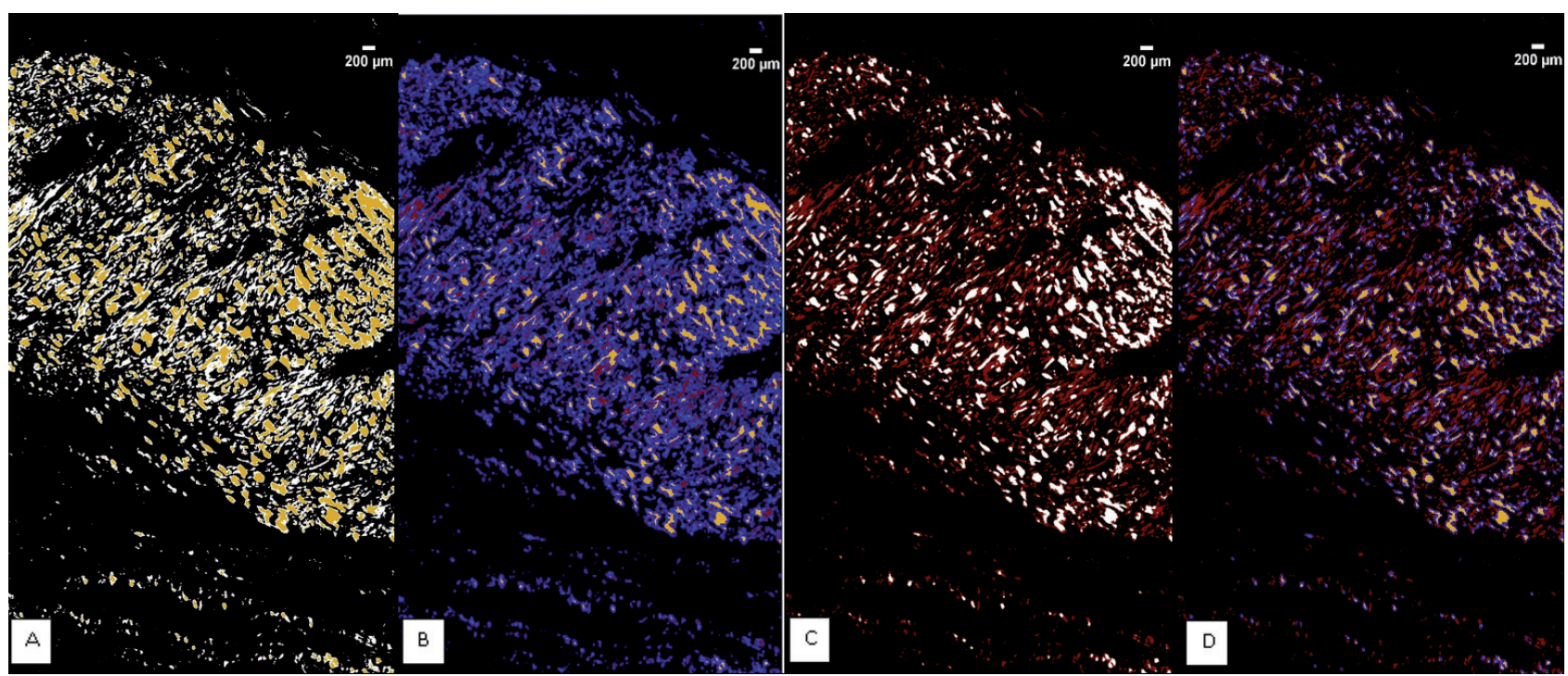

Figure 6. Photomicrographs of the healing area of skin wounds in diabetic mice treated with MSC. A \& B- Segmentation analysis for type 1 collagen. A- Cleavage in white for type 1 collagen fibres. B- Thresholding shown in blue for delimiting the perimeter of collagen 1, which is highlighted in red. C \& D- Segmentation analysis for type 3 collagen fibres. C- Targeting the white colour for identifying type 3 collagen. D- Thresholding shown in blue for delimiting the perimeter of type 3 collagen, which is highlighted in yellow. The threshold method was performed using the colour threshold programme in Image $\mathbf{J}^{\circledR}$. Picrosirius Red under polarised light [10x magnification]. 


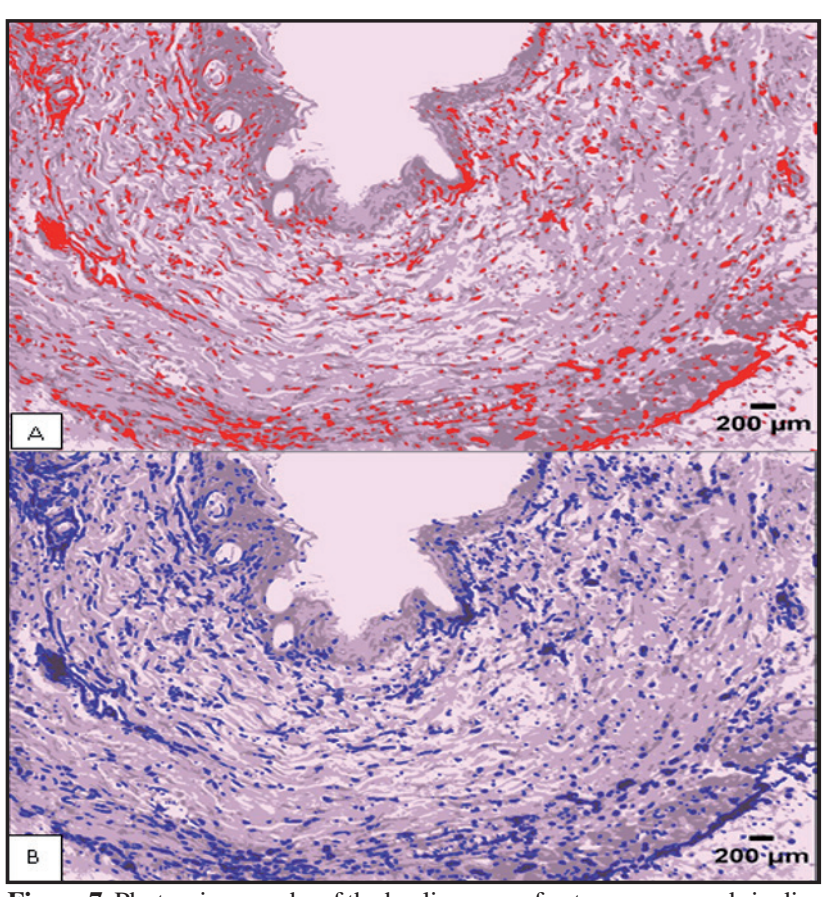

Figure 7. Photomicrographs of the healing area of cutaneous wounds in diabetic mice treated with MSC. A- Segmentation in red for the identification of elastic fibres. B- Thresholding shown in blue for delimiting the perimeter of the area of elastic fibres. The threshold method was performed using the colour threshold program in Image $\mathbf{J}^{\circledR}$. Weigert [10x magnification].

of neoelastogenesis since it intensified after maturation of the collagen fibres [27]. Therefore the data obtained for the elastic fibre volume in the present study may represent only one moment in time in the progression of the cutaneous cicatricial process. The higher deposition of elastic fibres in the skin lesions of the GCL and GME group, which presumably have lower collagenase levels than the GCT group, corroborate this theory.

Thus further longitudinal studies are needed to investigate the relationship between collagenase, elastogenesis and topical MSC therapy, as well as the effect of the MSC secretome on cutaneous healing, as described in a previous study [26].

The scientific literature describes the colour segmentation technique used in the present study in detail, including in several contemporary studies that have assessed the applicability of $\mathrm{K}$-means clustering in dermatological research $[5,4,12]$. This study opted for the target colour, as binarisation methods commonly employed in morphology can overestimate the values resulting from the absence of sample categorisation in different groups [18].

The morphometric method of colour segmentation reaffirmed the data obtained through stereology regarding the measurement of collagen fibres, thus only identifying significant differences for type 3 collagen fibres (ACOL3). Animals treated with autologous MSC showed the largest areas for this variable when compared to the other treatment groups (Table 2).

The positive contribution of dermatological therapy with autologous MSC was evidenced by the increased total surface of type 3 collagen (ACOL3) fibres and the space occupied by these fibres (VCOL3), thus undoubtedly eliminating possible misunderstandings about the behaviour of the variable. In subsequent studies, it will be necessary to investigate which molecular mechanisms precisely influence the genomics and proteomics of fibroblasts, as these cells permit the modulation of dermal collagenase in chronic wounds.

In a similar manner to that observed in elastic fibre volume, a smaller area of elastic fibres (AFE) was also identified among animals in the GCT group compared to the other treatment groups. Based on the equivalence of the statistical analysis between AFE and VEF and the brevity of time (12 days) used to evaluate the elastic fibre formation, the same biological hypotheses discussed previously for VEF are likely to persist; however, the colour segmentation technique has a low applicability for measuring elastic fibres in different tissues [12]. To date, no studies have been reported that apply this technique to the analysis of dermal elastic fibres. One recent study developed a specific algorithm for measuring elastic fibres in blood vessels [12], which indicates that this technique is still under development.

Although the data obtained for the area of elastic fibres were similar to the stereological method, it is necessary to develop algorithms to accurately measure the area of dermal elastic fibres, including to compare the data presented in this study.

\section{CONCLUSION}

Dermatological treatment of cutaneous wounds in diabetic mice using autogenous MSC induced a higher deposition of type 3 collagen fibres on the 12th day after the start of the cicatricial process, as demonstrated by an increased fibre area and volume. The increase in elastic fibre deposition was higher in animals with lower type 3 collagen fibre area and volume, thus demonstrating that there was no overlap of the stages of collagen and elastogenesis in the healing of chronic cutaneous wounds.

Correspondence was identified between the stereological and morphometric data obtained for the measurement of collagen and elastic fibres, thus 
demonstrating that the techniques are complementary and increase the accuracy of evaluating dermal fibres.

\section{MANUFACTURERS}

${ }^{1}$ Leica do Brasil Importação e Comércio Ltda. São Paulo, SP, Brazil. ${ }^{2}$ Olympus Corporation. New York, NY, USA.

${ }^{3}$ Sony Corporation. Tokyo, Japan.

${ }^{4}$ Positive Informatics. Curitiba, PR, Brazil.

${ }^{5}$ National Institute of Mental Health. Bethesda, MD, USA.
Ethical approval. The animal handling practices carried out prior to this study were approved by the Animal Experimentation Ethics Committee of the Federal University of Viçosa-MG according to the declaration $n^{\circ} 43 / 2007$ and the norms of the Brazilian College of Animal Experimentation.

Declaration of interest. The authors report no conflicts of interest. The authors alone are responsable for the contente and writing of paper.

\section{REFERENCES}

1 Amenábar J.M., Padilha D.M.P., Hugo F.N. \& Fossati A.C.M. 2003. The use of stereology as method in histological research. Revista da Faculdade de Odontologia de Porto Alegre. 44: 62-65.

2 Argôlo Neto N.M., Feitosa M.L.T., Sousa S.S., Fernandes P.B., Pessoa G.T., Bezerra D.O., Almeida H.M., Carvalho Y.K.P., Rocha A.R., Silva L.M.C. \& Carvalho M.A.M. 2016. Isolation, expansion, differentiation and growth kinetics essay in mesenchymal stem cells culture from the bone marrow of collared peccaries (Tayassu tajacu). Acta Scientiae Veterinariae. 44: 1-11.

3 Argôlo Neto N.M., Del Carlo R.J., Monteiro B.S., Nardi N.B., Chagastelles P.C., Brito A.F. \& Reis A.M. 2012. Role of autologous mesenchymal stem cells associated with platelet-rich plasma on healing of cutaneous wounds in diabetic mice. Clinical and Experimental Dermatology. 37: 544-553.

4 Bedoya S.A.O., Conceição L.G., Viloria M.I.V., Loures F.H., Valente F.L., Amorim R.L. \& Silva F.F. 2016. Characterization of types I and III collagen in the stroma of cutaneous squamous cell carcinoma in dogs. Arquivo Brasileiro de Medicina Veterinária e Zootecnia. 68: 147-154.

5 Brianezi G., Grandi F., Bagatin E., Mílvia Maria S. S., Enokihara M.M.S.S \& Miot H.A. 2015. Dermal type I collagen assessment by digital image analysis. Anais Brasileiros de Dermatologia. 90: 723-727.

6 Caskey R.C., Zgheib C., Morris M., Allukian M., Dorsett-Martin W., Xu J., Wu W. \& Liechty K.W. 2014. Dysregulation of collagen production in diabetes following recurrent skin injury: contribution to the development of a chronic wound. Wound Repair and Regeneration. 22: 515-520.

7 Charles-de-Sá L., Gontijo-de-Amorim N.F., Maeda Takiya C., Borojevic R., Benati D., Bernardi P. Sbarbati A. \& Rigotti G. 2015. Antiaging treatment of the facial skin by fat graft and adipose-derived stem cells. Plastic and Reconstructive Surgery. 135: 999-1009.

8 D'Ettorre M., Gniuli D., Iaconelli A., Massi G., Mingrone G. \& Bracaglia R. 2010. Wound healing process in post-bariatric patients: an experimental evaluation. Obesity Surgery. 20: 1552-1558.

9 Farghali H.A., AbdElKader N.A., Khattab M.S. \& AbuBakr H.O. 2017. Evaluation of subcutaneous infiltration of autologous platelet-rich plasma on skin-wound healing in dogs. Bioscience Reports. 10: 37.

10 Fritz K., Bernardy J., Tiplica G.S. \& Machovcova A. 2015. Efficacy of monopolar radiofrequency on skin collagen remodeling: a veterinary study. Dermatologic Therapy. 28: 122-125.

11 Han G. \& Ceilley R. 2017. Chronic Wound Healing: A Review of Current Management and Treatments. Advances in Therapy. 34: 599-610.

12 Hernández-Morera P., Travieso-González C.M., Castaño-González I., Mompeó-Corredera B. \& Ortega-Santana F. 2017. Segmentation of elastic fibres in images of vessel wall sections stained with Weigert's resorcin-fuchsin. Computer Methods and Programs Biomedicine. 14: 243-254.

13 Hocking A.M. 2012. Mesenchymal Stem Cell Therapy for Cutaneous Wounds. Advances in Wound Care. 1: 166-171.

14 Kamp S., Jemec G.B., Kemp K., Kjeldsen C.R., Stenderup K., Pakkenberg B. \& Dam T.M. 2009. Application of stereology to dermatological research. Experimental dermatology. 18: 1001-1009.

15 Kim S.M., Kim Y.H., Jun Y.J., Yoo G. \& Rhie J.W. 2016. The effect of diabetes on thewound healing potential of adipose-tissue derived stem cells. International Wound Journal. 13: 33-34.

16 Lewis P.N., White T.L., Young R.D., Bell J.S., Winlove C.P. \& Meek K.M. 2016. Three-dimensional arrangement of elastic fibers in the human corneal stroma. Experimental Eye Research. 146: 43-53. 
17 Marcos-Garcés V., Harvat M., Molina Aguilar P., Ferrández Izquierdo A. \& Ruiz-Saurí A. 2017. Comparative measurement of collagen bundle orientation by Fourier analysis and semiquantitative evaluation: reliability and agreement in Masson's trichrome, Picrosirius red and confocal microscopy techniques. Journal of Microscopy. 267: 130-142.

18 Miot H.A. \& Brianezi G. 2010. Morphometric analysis of dermal collagen by color clusters segmentation. Anais Brasileiros de Dermatologia. 85: 361-364.

19 Nuschke A. 2013. Activity of mesenchymal stem cells in therapies for chronic skin wound healing. Organogenesis. 10: 29-37.

20 Otero-Viñas M. \& Falanga V. 2016. Mesenchymal Stem Cells in Chronic Wounds: The Spectrum from Basic to Advanced Therapy. Advanced in Wound Care. 5: 149-163.

21 Ramos H.V., Simões M.J., Pontes P.A., Neves L.R., Barbosa L.H., De Biase N.G. \& Oshima C.T. 2005. Immunohistochemistry as a method to study elastic fibers of human vocal fold. Brazilian Journal of Otorhinolaryngology. 71: 486-491.

22 Reinke J.M. \& Sorg H. 2012. Wound Repair and Regeneration. European Surgical Research. 49: 35-43.

23 Shin M.K., Lee J.W., Kim Y.I., Kim Y.O., Seok H. \& Kim N.I. 2014. The effects of platelet-rich clot releasate on the expression of MMP-1 and type I collagen in human adult dermal fibroblasts: PRP is a stronger MMP-1 stimulator. Molecular Biology Reports. 41: 3-8.

24 Sivaraman B., Bashur C. A. \& Ramamurthi A. 2012. Advances in biomimetic regeneration of elastic matrix structures. Drug Delivery and Translational Research. 2: 323-350.

25 Smolle J. 2000. Computer recognition of skin structures using discriminant and cluster analysis. Skin Research and Technology. 6: 58-63

26 Swaminathan G., Sivaramam B. \& Ramamurthi A. 2016. Biomolecular Regulation of Elastic Matrize Regeneration and Repair. In: Ramamurthi A. \& Kothapalli C. (Eds). Elastic Fiber Matrices Biomimetic. Approaches to Regeneration and Repair. Oklahoma. Boca Raton: CRC Press - Taylor \& Francis Group, pp.118-144.

27 Witte M.B. \& Barbul A. 1997. General principles of wound healing. The Surgery Clinics of North America. 77: 509528 .

28 Xue S. \& Li L. 2011. Upregulation of collagen type 1 in aged murine dermis after transplantation of dermal multipotent cells. Clinical and Experimental Dermatology. 36: 775-781.

29 Yamawaki-Ogata A., Hashizume R., Fu X.M., Usui A. \& Narita Y. 2014. Mesenchymal stem cells for treatment of aortic aneurysms. World Journal of Stem Cells. 26: 278-287

30 Zhang W.G., He L., Shi X.M., Wu S.S., Zhang B., Mei L., Xu Y.J., Zhang Z.X., Zhao J.P. \& Zhang H.L. 2014. Regulation of transplanted mesenchymal stem cells by the lung progenitor niche in rats with chronic obstructive pulmonary disease. Respiratory Research. 25: 15-33.

31 Zhao P., Schulz T.C., Sherrer E.S., Weatherly D.B., Robins A.J. \& Wells L. 2015. The human embryonic stem cell proteome revealed by multidimensional fractionation followed by tandem mass spectrometry. Proteomics. 15: 554-566. 